\title{
Fabricación del acero de Damasco: estudio metalográfico ${ }^{(\bullet)}$
}

\author{
A.J. Criado ${ }^{(*)}$, J.A. Martínez ${ }^{(*)}$, R. Calabrés ${ }^{(*)}$ y D. Arias ${ }^{(*)}$ \\ Resumen Acero de Damasco es la denominación que los europeos dieron al material del que estaban hechas las \\ espadas musulmanas durante la época de las Cruzadas. Este acero hipereutectoide, presenta un alto \\ contenido de carbono que supera en todos los casos el $0,8 \%$, llegando en algunos casos al $2,1 \%$ en \\ masa. El secreto de sus buenas características mecánicas radica en los procesos de forja en caliente, \\ en un intervalo de temperaturas entre 650 y $850^{\circ} \mathrm{C}$, a los que se puede someter. El temple final en \\ agua, salmuera u otras soluciones acuosas, confiere a las armas fabricadas con este acero una buena \\ resistencia en su filo cortante y una tenacidad elevada. En la presente investigación se estudian los \\ procesos de fabricación de este tipo de acero. Se ha aplicado la microscopía electrónica de barrido al \\ estudio de materiales fabricados por los autores siguiendo los métodos artesanos antiguos de forja y \\ temple.
}

Palabras clave: Acero hipereutectoide. Metalografía. Acero de Damasco. Forja en caliente. Fabricación.

\section{Manufacture of Damascus steel: Metallographical study}

\begin{abstract}
Damascus Steel is the denomination that the Europeans gave to the material with which the musulman swords were manufactured during the Era of the Crusades. This hypereutectoid steel presents a high content in carbon, more than $0.8 \%$, and in some cases up to $2 \%$ in weight. The secret of its good mechanical characteristics is based in the hot forging process in the temperatures interval between 650 and $850{ }^{\circ} \mathrm{C}$. The final quenching in water, brine or other aqueous solutions, confers to the swords manufactured with this steel a good resistance to its cutting edge and a high toughness. In the present investigation, the manufacture processes of this type of steel are studied. Electronic scanning microscopy has been applied to the study of materials manufactured by the authors following the ancient craftsmen methods of forging and quenching.
\end{abstract}

Keywords: Hypereutectoid steel. Metallography. Damascus Steel. Hot forging. Manufacturing.

\section{INTRODUCCIÓN}

Son muchas las contribuciones que Córdoba aportó al Occidente Europeo durante la época musulmana. Muchas reliquias de aquel pasado glorioso del Emirato y del Califato están aún presentes en la actualidad. Algunas, como la Mezquita, son patrimonio de la Humanidad. La ciencia y la cultura florecieron como no lo habían hecho desde el Imperio Romano.

Sin embargo, pocos saben, o reconocen, que el corazón de aquella Córdoba esplendorosa latía gra-

(•) Trabajo recibido el día 30 de octubre de 1995.

(*) Dpto. de Ciencia de los Materiales e Ingeniería Metalúrgica. Facultad de Ciencias Químicas. Universidad Complutense de Madrid. 28040-Madrid (España). cias al acero de sus espadas y, menos aún, que ese duro metal era un famosísimo acero. La fuerza de las armas cordobesas contribuyó al sostenimiento de todo aquel mundo maravilloso (1).

De Damasco no sólo vino el Sacre Omeya, aquel primer Abderrahman que trajo a estas tierras todo aquello que le consolaba de la pérdida inexorable de su familia y sus hermosos palacios de Siria, sino también, en los sables de sus valerosos guerreros, el acero mejor templado del mundo.

Este formidable acero damasquino, traído a España por los árabes, fue de nuevo conocido por los europeos en la época de las cruzadas, donde lo probaron en su propia carne y lo bautizaron como Acero de Damasco (2).

Es un acero con propiedades mecánicas fuera de lo corriente. Se trata de aceros con contenidos de 
carbono muy elevados que los convierten en materiales duros y resistentes al desgaste. Su filo permanece inalterable y extraordinariamente cortante por continuado y severo uso que se haga de él. Su único posible defecto, la extremada fragilidad, la corregían los herreros con una combinación de forja, a la temperatura conveniente, y temple controlado.

Córdoba, en la época musulmana, lo introdujo en España junto con el arte de su forja. Esto supuso el comienzo de una noble tradición en la fabricación de armas de acero que dieron prestigio a esta nación (3).

En esta investigación se han utilizado aceros con un contenido del 1,4 al 1,6\% C. Este tipo de aceros pertenece a la familia de los hipereutectoides extraduros.

En este trabajo se han intentado reproducir todas las etapas del proceso de fabricación de las espadas realizadas con acero de Damasco al más puro y legítimo estilo medieval según la literatura consultada (Figs. 1 y 2) (4-16).

Se ha utilizado la forja manual, y todos los tratamientos mecánicos y térmicos los ha llevado a cabo al estilo antiguo Juan Pozón, herrero tradicional de Cerro Muriano (Córdoba), fabricante de rejas, cancelas y piezas y objetos muy diversos forjados en hierro y acero (4).

Los cambios microestructurales resultantes de estos procesos se han observado mediante microscopía electrónica de barrido (MEB).

\section{TÉCNICA EXPERIMENTAL}

Se han realizado experiencias preliminares de cara a obtener el material base, es decir, el acero hipereutectoide, según los métodos habitualmente utilizados por aquellos herreros artesanos (Fig. 1).

Los métodos para alcanzar la composición con un contenido entre 1,4 y $1,6 \% \mathrm{C}$ consisten en partir de hierro puro y carburarlo, o bien partir de fundición blanca y descarburar hasta la composición adecuada.

Los ensayos realizados para la obtención del acero de Damasco, siguiendo ambos métodos, han probado que las dos posibilidades parecen ser los caminos seguidos para llegar a obtener la composición de dichos aceros de Damasco.

Sin embargo, el objetivo principal de este trabajo era el seguimiento de los tratamientos termomecánicos en los aceros de Damasco, y no la forma de lograr la adecuada composición. Debido a la necesidad de disponer siempre de una calidad uniforme para toda la investigación, se recurrió a utilizar aceros comerciales de composición idéntica a los obtenidos en nuestras pruebas de reproducción de la materia prima al más puro estilo de Damasco. Estos aceros se fabrican comercialmente bajo la denominación F-516 y F-517.

La conformación plástica se realizó por forja en caliente en el intervalo de temperaturas de 650 a $850{ }^{\circ} \mathrm{C}$. El comportamiento superplástico que presentan dichos aceros a esta temperatura permite

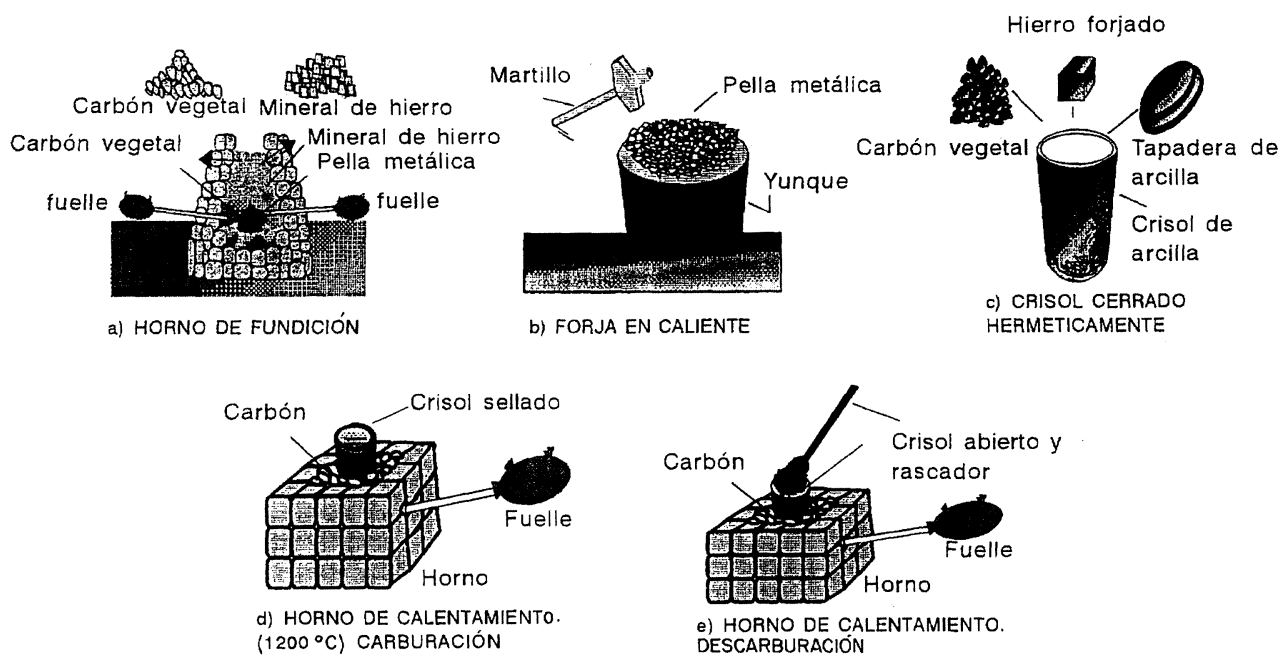

FIG. 1.- Proceso completo seguido en la época medieval para la fabricación del acero de Damasco: a) Reducción de los óxidos de hierro (mineral) con carbón vegetal. b) Forja de la pella de hierro. c) y d) Carburación del hierro forjado a partir de la pella. e) Descarburación de hierro fundido en el caso de haberlo obtenido en la primera etapa.

FIG. 1.- Complete process followed in the Medieval Age for the obtention of Damascus Steel (hypereutectoidic steel): a) Reduction of the iron oxides (mineral) with vegetable coal. b) Forging of the iron pellet. c) and d) Carburizing of the wrought iron from the pellet. e) Uncarburizing of molten iron obtained in the first stage. 
triturar la cementita proeutectoide situada en los límites de grano austeníticos (2, 7-9, 14 y 15). La tecnología utilizada en la presente investigación es la misma que la de los herreros antiguos; mediante una fragua de carbón y un fuelle accionado por una soplante, único artilugio moderno de la instalación. La forja se realizó sobre yunque con un martillo de algo más de $1 \mathrm{~kg}$ de peso (Fig. 2).

El herrero realizó el proceso de forja llevando la cadencia de los golpes bajo el compás de cantes flamencos. Esta manera de medir el tiempo y el ritmo de la forja permite un control bastante exacto del proceso, y parece ser una milenaria característica propia de estos artesanos. La temperatura de forja se controló mediante el color del metal en el intervalo entre el rojo cereza y el rojo sangre. Con el fin de asegurar un valor de temperatura exacto, se utilizó un medidor de temperaturas consistente en un termopar de contacto tipo Chromel-Alumel en vaina de acero inoxidable.

Partiendo de barra de $20 \mathrm{~mm}$ de diámetro se forjaron probetas prismáticas de $20 \times 4 \mathrm{~mm}$ de sección y $50 \mathrm{~mm}$ de largo con diferentes grados de reducción, llegando ésta a alcanzar el $75 \%$ en algunos casos (Fig. 3c).

Con el fin de comprobar las dificultades asociadas a la forja de una pieza real, se forjó también un puñal (Fig. 3a).

Para alcanzar el endurecimiento final de las probetas, tajadera y puñal, se realizó un temple posterior en agua. La severidad del temple se ajustó realizando inmersiones sucesivas y agitación en el agua a tem-

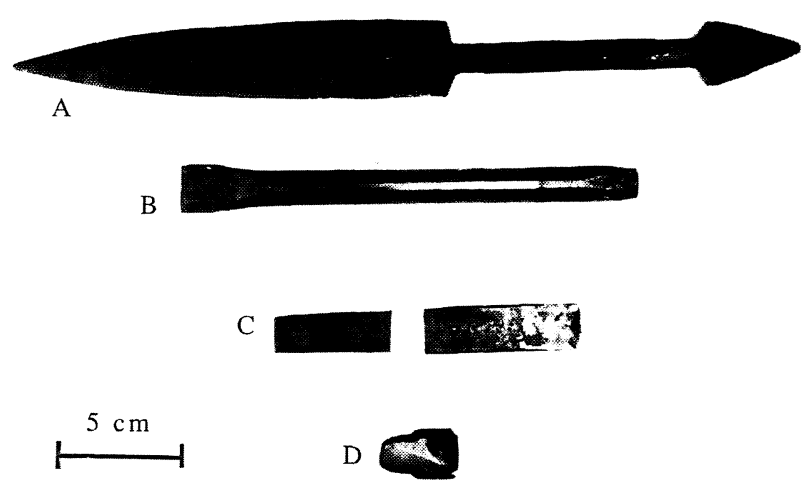

FIG. 3.- a) Daga forjada según el método medieval con acero de Damasco (1,4-1,6 \% C). b) Tajadera fabricada de la misma forma que la daga para comprobar las características de tenacidad, dureza y fragilidad del filo. c) Probetas fabricadas con el mismo acero y con los mismos procedimientos para el estudio metalográfico. d) Trozo de acero de Damasco obtenido por carburación de hierro.

FIG. 3.- a) Dagger forged with steel of Damascus (1.4-1.6 \% C) according with the medieval method. b) Chopper manufactured in the same way as the dagger in order to study the characteristics of toughness, hardness and fragility of the edge. c) Test sample manufactured with the same procedures for the metallographic study. d) Piece of

Damascus Steel obtained by carburizing of iron.

peratura ambiente durante $5 \mathrm{~s}$, siguiendo el sonido provocado durante el enfriamiento al más puro estilo

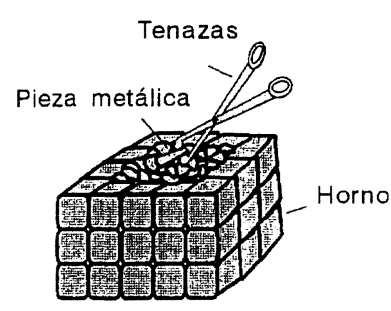

a) CALENTAMIENTO $650-850^{\circ} \mathrm{C}$

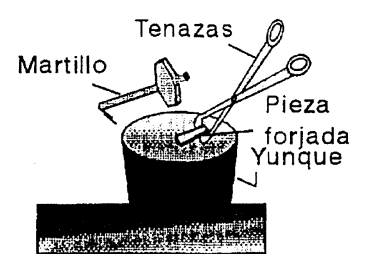

b) FORJA EN CALIENTE

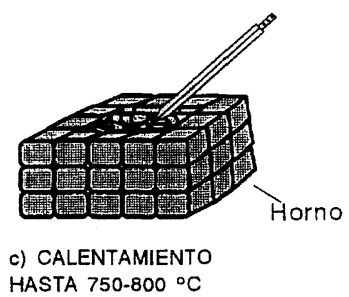

HASTA $750-800^{\circ} \mathrm{C}$

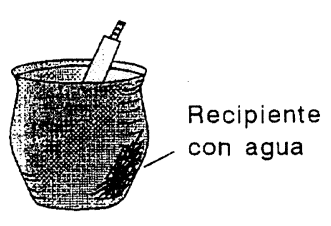

d) OPERACIÓN DE TEMPLE

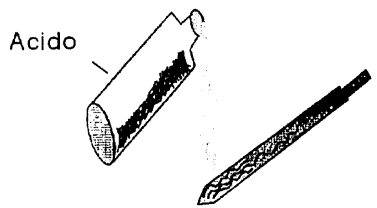

e) GRABADO QUIMICO CON ÁCIDO

FIG. 2.- Proceso completo seguido en la época medieval para la fabricación de espadas con acero de Damasco: a) y b) Forja en caliente en el intervalo de 650 a $850{ }^{\circ} \mathrm{C}$. c) Calentamiento hasta $750-800{ }^{\circ} \mathrm{C}$. d) Temple controlado en agua. e) Grabado químico con ácido.

FIG. 2.- Complete process followed in the Medieval Age for the manufacture of swords in the Damascus Steel: a) and b) Hot forging in the temperatures interval from 650 to $850{ }^{\circ} \mathrm{C}$. c) Warming up to $750-800{ }^{\circ} \mathrm{C}$. d) Controlled quenching in water. e) Chemical etching with acid. 
tradicional y legendario de los herreros cordobeses actuales. Se observó la aparición de un tono azulado en la superficie hasta su enfriamiento total.

La comprobación de ausencia de fragilidad y fuerza de corte en el filo se realizó con una herramienta gemela en forma de tajadera. Las buenas características del filo se comprobaron observando el comportamiento ante el corte de una plancha de acero suave normalizado. De esta forma se llegó a la selección del temple más adecuado a las características requeridas.

El seguimiento de las características mecánicas alcanzadas en cada operación se realizó mediante ensayos de Dureza Rockwell C con un durómetro Equotip provisto de sondas tipo D y C. Las medidas de dureza no sólo tenían como objetivo controlar las diferentes etapas de fabricación, sino también asegurar la igualdad en cuanto a las características estructurales y mecánicas de las diferentes piezas fabricadas, daga, tajadera y demás probetas.

Las probetas forjadas se prepararon para su observación metalográfica mediante MEB. El ataque se realizó con Nital modificado al $45 \%$ en masa durante $15 \mathrm{~s}$.

\section{RESULTADOS Y DISCUSIÓN}

El objetivo de los herreros fabricantes de este acero era conseguir un filo cortante resistente al desgaste y a los golpes sin que esta dureza trajese aparejada la fragilidad.

La utilización de aceros extraduros con contenidos de carbono comprendidos entre el 1,3 y el 2,1 $\%$ en masa (7-9) reunía estas características de dureza y resistencia al desgaste; sin embargo, sólo si la conformación plástica por forja en caliente se hacía correctamente se evitaba la fragilidad (Fig. 2), obteniéndose, al mismo tiempo, una gran tenacidad.

El éxito fundamental de los herreros que fabricaban armas con el acero de Damasco (11 y 12) estaba en la forja. Resultaba vital, sobre todo, la elección del intervalo de temperaturas en que se efectuaba esta operación, que debía estar comprendido entre 650 y $850^{\circ} \mathrm{C}$ (Fig. 4), es decir, entre el rojo sangre y el cereza. En este intervalo el comportamiento superplástico de estos aceros permite una excelente conformación, sin provocar consecuencias mecánicas negativas, originando la trituración de la cementita proeutectoide del límite de grano austenítico que se dispersa en la matriz del acero formando bandas en la dirección de forja (Figs. 5, 6 y 7).

La fragilidad desaparece y aumenta la tenacidad, manteniendo una excelente resistencia debida al elevado contenido de carbono y, por tanto, un filo cortante a pesar de un uso muy severo.

El trabajo en caliente permite una forja correcta

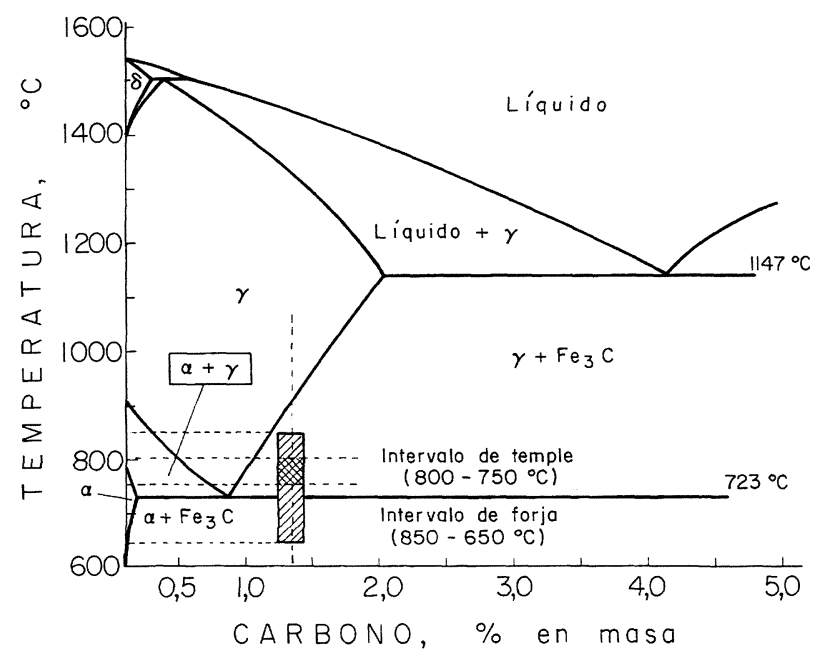

FIG. 4.- Diagrama del sistema Fe-C en el que se han representado los intervalos de temperatura utilizados en la investigación.

FIG. 4.- Phases diagram of the Fe-C system showing the suitable temperature intervals for hot forging and quenching used in the investigation.

hasta conseguir la forma deseada, siempre que se realice en el intervalo de temperaturas adecuado, verdadero secreto del acero de Damasco. Los europeos, acostumbrados a contenidos más bajos de carbono, solían trabajar en caliente a temperaturas más elevadas. Cuanto más elevadas eran las temperaturas, mayor plasticidad encontraban. Esta fue, posiblemente, la razón por la que no dieron con el secreto de los aceros de Damasco (2), el trabajo

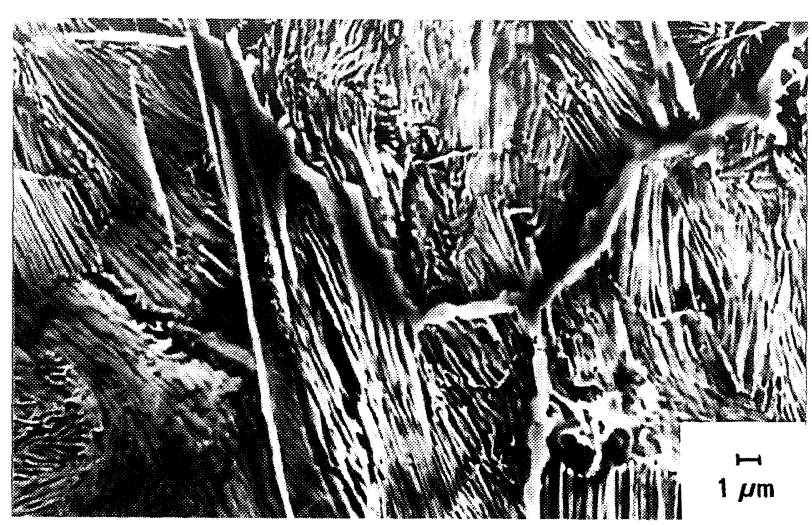

FIG. 5.- Estructura típica de un acero de Damasco $1,4 \% \mathrm{C}$ normalizado. Cementita proeutectoide en límite de grano y cementita acicular con estructura Widmänsttaten. Ataque: Nital modificado al $45 \%$, $1 \mathrm{~min}$.

FIG. 5.- Typical structure of a normalized Damascus Steel (1.4\% C). Proeutectoidic cementite at grain boundaries and Widmänsttaten needled cementite. Etching: modified Nital $45 \%, 1 \mathrm{~min}$. 


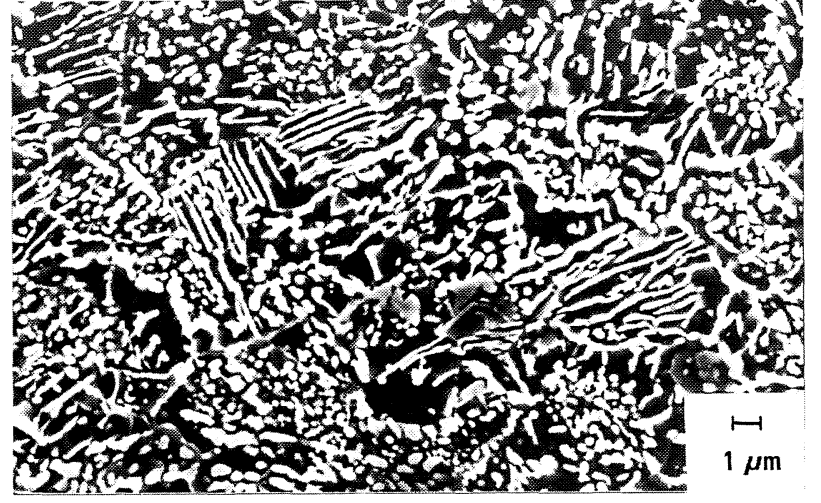

FIG. 6.- Estructura del acero de Damasco forjado. Cementita proeutectoide triturada y repartida en una matriz ferrítica y de cementita eutectoide esferoidizada. Ataque: Nital modificado al 45\%, 1 min.

FIG. 6.- Structure of a hot forged Damascus Steel. Spheroidized proeutectoidic cementite, distributed in a matrix of ferrite and spheroidized eutectoidic cementite. Etching: modified Nital 45 \%, 1 min.

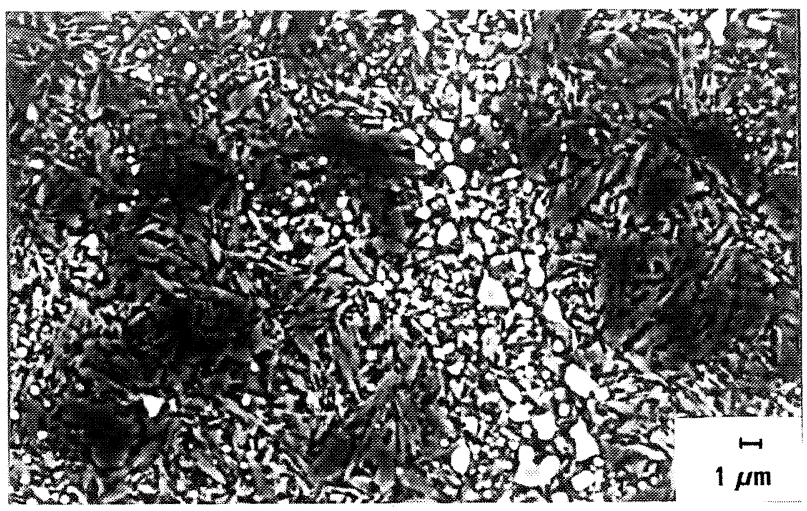

FIG. 7.- Estructura del acero de Damasco templado. Cementita proeutectoide esferoidizada distribuida en bandas en una matriz martensítica. Ataque: Nital modificado al $45 \%, 1 \mathrm{~min}$.

FIG. 7.- Structure of the quenched Damascus Steel. Spheroidized proeutectoidic cementite, distributed in bands in a martensitic matrix. Etching: modified Nital $45 \%, 1$ min.

a temperaturas relativamente bajas, y que no fue descubierto hasta el siglo XIX.

Una vez conseguidas unas excelentes características de resistencia y tenacidad durante la forja, la resistencia puede mejorarse aún mediante adecuadas operaciones de temple.

Durante la operación de temple no se debe calentar por encima de la temperatura en la que el acero se sitúa en el campo austenítico, ya que ésto provoca la redisolución de la cementita proeutectoide localizada en forma de bandas, algo que tiene consecuencias muy negativas en las características mecánicas del acero templado. En algunos casos, los frágiles límites de grano austenítico saturados de cementita proeutectoide se pueden regenerar de nuevo.

Durante la operación de forja en caliente ocurren diferentes cambios estructurales. Los carburos correspondientes a la cementita proeutectoide triturada, dispersada y alineada en bandas durante la forja en caliente, aparecen muy esferoidizados (Fig. 7). Este hecho tiene influencias positivas en la tenacidad del material (7-9).

En la presente investigación, para los contenidos de carbono utilizados, el calentamiento, de apenas 5 min antes del temple, se efectuó en el intervalo de temperaturas de 750 a $800{ }^{\circ} \mathrm{C}$ (Fig. 4).

El temple se realizó en agua obteniéndose una matriz martensítica con bandas de carburos en la dirección de forja (Fig. 7), alcanzándose una dureza de 65-67 HRC (832-900 HV).

El aspecto de estrías serpenteantes de las armas fabricadas así aparecía por el pulido final de la superficie y su ataque con jugos ácidos naturales (limón, vinagre, etc...) que revelaba la estructura en bandas de la cementita proeutectoide en forma de estrías de color blanquecino; aunque no siempre es símbolo de la máxima calidad en la fabricación de estos aceros ( 2 y 7-9), siendo su presencia más evidente para contenidos de carbono muy elevados.

El aprendizaje de los herreros andalusíes en la transformación de la estructura frágil de estos aceros en otra más resistente y tenaz (Fig. 8), durante las etapas del Emirato y Califato de Córdoba, hizo que estas tecnologías de forja en caliente a temperaturas bajas -rojo cereza y rojo sangre- de aceros de alto contenido de carbono (1,4 a 2,1\% C) (7-9) pasarán a ser la tecnología propia de los herreros españoles. No ha sido, por tanto, algo desconocido para España desde entonces la preparación de armas con aceros de gran resistencia y tenacidad.

\section{CONCLUSIONES}

- Ha quedado patente que la etapa crítica en la fabricación de armas con estos aceros está en que su forja en caliente debe realizarse en el intervalo de temperaturas de 650 a $850{ }^{\circ} \mathrm{C}$ (rojo cereza y rojo sangre), para triturar la cementita proeutectoide situada en los límites de grano distribuidos en la matriz del acero sin permitir la regeneración de la estructura perjudicial original.

- El temple en agua después de un breve calentamiento de $5 \mathrm{~min}$ a temperaturas comprendidas entre los $750-800{ }^{\circ} \mathrm{C}$, produce una estructura martensítica muy dura con la cementita proeutectoide con morfologías esferoidizadas distribuida en bandas en la dirección de forja.

- La severidad del temple influye en la tenacidad final del acero, para lo que es necesario jugar 


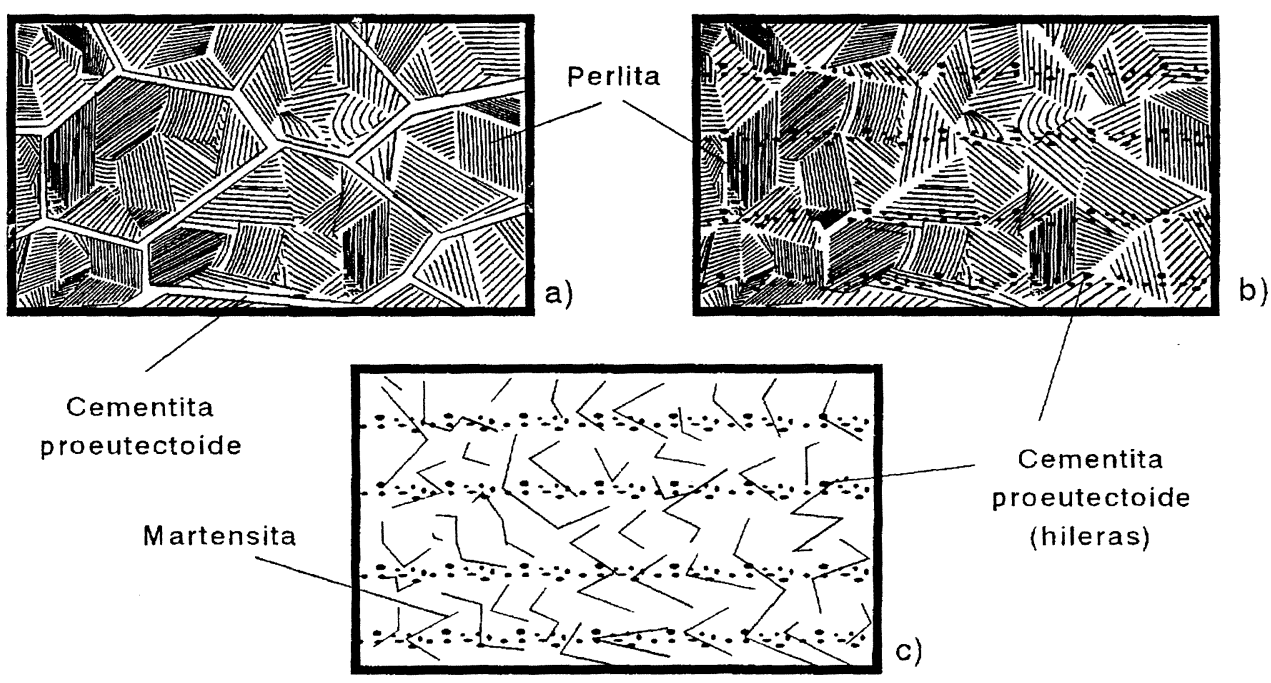

FIG. 8.- Representación esquemática de los cambios microestructurales producidos en los aceros de Damasco durante la fabricación de las espadas: a) Acero Fe-1,4\% C laminado en caliente y normalizado. Cementita proeutectoide precipitada en límite de grano. b) Acero forjado en caliente $\left(650-850{ }^{\circ} \mathrm{C}\right)$ y normalizado. Cementita proeutectoide triturada y esferoidizada redistribuida en bandas en la matriz perlítica. c) Acero forjado en caliente $\left(650-850^{\circ} \mathrm{C}\right)$ y templado en agua (desde $\left.750-800^{\circ} \mathrm{C}\right)$. Estructura de temple con la cementita proeutectoide esferoidizada en bandas en una matriz martensítica.

FIG. 8.- Schematic representation of the microstructural changes produced in the Damascus Steels during the manufacture of swords: a) Fe-1.4 \% C hot rolled and normalized steel. Proeutectoidic cementite at grain boundaries. b) Hot forged (650-850 ${ }^{\circ} \mathrm{C}$ ) and normalized steel. Proeutectoidic cementite, disintegrated, spheroidized and distributed in bands in the perlitic matrix. c) Hot forged (650-850 $\left.{ }^{\circ} \mathrm{C}\right)$ and quenched in water from $750-800^{\circ} \mathrm{C}$. Spheroidized proeutectoidic cementite in bands in a martensitic matrix.

con el medio templante y con las velocidades de inmersión en el mismo, que dependen de la masa de acero y sección del arma. Para estos aceros, y de forma artesanal para el tamaño del puñal fabricado, se debe templar en agua a temperatura ambiente, durante $5 \mathrm{~s}$, sacarlo fuera del agua y esperar hasta la aparición de un color azulado característico, después volver a introducirlo en el agua hasta su enfriamiento total.

- El pulido final de la superficie de las piezas fabricadas y su ataque con jugos ácidos naturales permite revelar las estrías serpenteantes blanquecinas características de este acero; aunque la presencia de éstas no es siempre un símbolo de la máxima calidad.

\section{Agradecimiento}

A Juan Pozón Jilarte, herrero tradicional de Cerro Muriano (Córdoba). Al Museo del Ejército (Madrid), por permitir el acceso a sus fondos.

\section{REFERENCIAS}

(1) Lévi Provençal, E. y Torres Balbas, L. Historia de España: España Musulmana (Tomos I y II). Dirigida por
D. Ramón Menéndez Pidal. Ed. Espasa-Calpe, S.A. Madrid, 1987.

(2) Sherby O.D. y Wadsworth, J. Invest. Cienc., Abr., 1985: 76-82.

(3) CAlvo, F.A. La España de los Metales. CENIM. Madrid, 1964.

(4) Criado, A.J. Córdoba y el Acero de Damasco. Diario de Córdoba, S.A. Mar., 1995.

(5) PAuseri, C. Gladius, IV, 1965: 5-66.

(6) Piaskowsky, J. J. Hist. Arabic Sci., 2, 1988: 3-30.

(7) Verhoeven, J.D. Metallography, 20, 1987: 145-151.

(8) Verhoeven, J.D. y Jones, L.L. Metallography, 20, 1987: 153-180.

(9) Verhoeven, J.D., Baker, H.H., Peterson, D.T., Clark, H.F. y JATES, W.M. Mat. Char., 24, 1990: 205-227.

(10) Sachse, M. NW, Verl. für Nene Wiss. Bremerhaven, 1989: 84.

(11) Wadsworth, J. y Sherby, O.D. Mat. Char., 28, 1992: 165-172.

(12) Verhoven, J.D. y Peterson, D.T. Mat. Char., 29, 1992: 335-341.

(13) Lacey, B. y Brooks, C.R. Mat. Char., 29, 1992: 243248.

(14) Wadsworth, J. y Sherby, O.D. Prog. Mat. Sci., 25, 1980: 35-68.

(15) Sherby, O.D., Oyama, T., Kum, D.W., Walser, B. y WADSWORTH, J. J. Met., 37, 1985: 50-56.

(16) Sherby. O.D. y Wadsworth, J. Sci. Amer. Lett., 252, 1985: 7. 